Pacific Journal of Mathematics

ON REPRESENTING ANALYTIC GROUPS WITH THEIR 


\title{
ON REPRESENTING ANALYTIC GROUPS WITH THEIR AUTOMORPHISMS
}

\section{G. HOCHSCHILD}

\begin{abstract}
A real or complex Lie group is said to be faithfully representable if it has a faithful finite-dimensional analytic representation. Let $G$ be a real or complex analytic group, and let $A$ denote the group of all analytic automorphisms of $G$, endowed with its natural structure of a real or complex Lie group. The natural semidirect product $G \rtimes A$ is a real or complex Lie group, sometimes called the holomorph of $G$. We show that if $G$ is faithfully representable and if the maximum nilpotent normal analytic subgroup of $G$ is simply connected then $G \rtimes A$ is faithfully representable.
\end{abstract}

This result follows quite easily from well-known representationtheoretical results and techniques. What we use is contained in [1, Ch. XVIII], and all the references given below are to this. Nominally, these references cover only the real case. However, as explained loc. cit., both the results and their proofs are almost identical in the complex case.

Thanks are due to Martin Moskowitz who drew my attention to this question and who 'obtained a number of special results that are consequences of the theorem below and contain suggestions for its proof.

Proposition. Let $G$ be a faithfully representable real or complex analytic group, and let $N$ be the maximum nilpotent normal analytic subgroup of $G$. If $N$ is simply connected there is a faithful finite-dimensional analytic representation of $G$ whose restriction to $N$ is unipotent.

Proof. By Theorem 4.3 (or 4.7), $G$ is a semidirect product $B \rtimes H$, where $B$ is solvable and simply connected, and $H$ is reductive. The construction of $B$ is carried out in the proof of Theorem 4.2, and this shows that, if $N$ is simply connected, one can arrange to have $N \subset B$ (one begins with a semidirect product decomposition of the radical of $G$ having the form $M \rtimes Q$, where $Q$ is reductive, and $M$ is simply connected and contains $N$ ).

By Theorem 3.1, there exists a faithful finite-dimensional analytic representation $\rho$ of $B$ whose restriction to $N$ is unipotent. Now $\rho$ satisfies the conditions of Theorem 2.2, so that (enlarging the representation space of $\rho$ ) one can extend $\rho$ to a finite-dimensional 
analytic representation of $G$ whose restriction to $B$ is faithful and whose restriction to $N$ is still unipotent. Any given faithful finitedimensional analytic representation of $G$, via restriction to $H$ and the canonical homomorphism $G \rightarrow H$, yields a finite-dimensional analytic representation of $G$ whose kernel is precisely $B$. The direct sum of this and the representation obtained above satisfies the requirements of the proposition.

THEOREM. Let $G$ be a faithfully representable real or complex analytic group. Let $N$ be the maximum nilpotent normal analytic subgroup of $G$, and let $A$ be the group of all analytic automorphisms of $G$. If $N$ is simply connected then $G \rtimes A$ is faithfully representable.

Proof. Let $B$ denote the group of all Lie algebra automorphisms of the Lie algebra $\mathscr{L}(G)$ of $G$. This is an algebraic linear group, and we denote its algebraic identity component by $B_{1}$. The Lie algebra of $B_{1}$ may be identified with the Lie algebra of all derivations of $\mathscr{L}(G)$. If $R$ is the radical of $G$ then $\mathscr{L}(R)$ is the radical of $\mathscr{L}(G)$, while $\mathscr{L}(N)$ is the maximum nilpotent ideal of $\mathscr{P}(G)$. Therefore, by a well-known result from Lie algebra theory, every derivation of $\mathscr{L}(G)$ sends $\mathscr{L}(R)$ into $\mathscr{L}(N)$. This implies that $B_{1}$ acts trivially on $\mathscr{L}(R) / \mathscr{L}(N)$.

Let $A^{\circ}$ denote the group of all elements of $A$ whose canonical images in $B$ belong to $B_{1}$. Then $A^{\circ}$ acts trivially on $R / N$. Since $B_{1}$ is normal and of finite index in $B$, the group $A^{\circ}$ is normal and of finite index in $A$.

Let $I$ denote the group of inner automorphisms of $G$. Clearly, $I \subset A^{\circ}$. Now let $S$ be a maximal semisimple analytic subgroup of $G$, and let $T$ be the subgroup of $A^{\circ}$ consisting of the elements of $A^{\circ}$ that keep the elements of $S$ fixed. Since every maximal semisimple analytic subgroup of $G$ is a $G$-conjugate of $S$, every coset of $I$ in $A$ contains an element that stabilizes $S$. It follows from this that the group $T I$ is normal in $A^{\circ}$. Since the group of inner automorphisms of $S$ is of finite index in the group of all analytic automorphisms of $S$, it follows also that $T I$ is of finite index in $A^{\circ}$.

Clearly, $A^{\circ}$ contains the identity component $A_{1}$ of $A$. If $n$ is the index of $T I$ in $A^{\circ}$ then $a^{n}$ belongs to $T I$ for every $a$ in $A_{1}$. Since $A_{1}$ is an analytic group, these elements $a^{n}$ generate $A_{1}$. Hence $A_{1} \subset T I$. We conclude that $T I$ is open and of finite index in $A$.

Now let $\rho$ be a representation of $G$ with the properties stated in the proposition. Let $V$ denote the space of representative functions on $G$ that are associated with $\rho$. Then $V$ is finite-dimensional and stable under the right and left translation actions of $G$. If $\rho^{\prime}$ 
is the semisimple representation of $G$ that is associated with $\rho$ then $N$ is contained in the kernel of $\rho^{\prime}$. Let $x$ be an element of the radical of $G$, and let $\tau$ be an element of $T$. Then $\tau(x) x^{-1} \in N$, so that $\rho^{\prime}\left(\tau(x) x^{-1}\right)$ is the identity map on the representation space. Since the elements of $T$ keep the elements of $S$ fixed, it is now clear that Lemma 2.1 applies to our present situation and gives the result that the space $V \circ T$ spanned by the composites of the elements of $V$ with those of $T$ is still finite-dimensional. From the 2-sided $G$-stability of $V$, it is clear that $V \circ(T I)=V \circ T$.

Let us write $J$ for $T I$, and $W$ for $V \circ T$. We define an action of $G \rtimes J$ on $W$ by setting $(x, \alpha) \cdot w=x \cdot\left(w \circ \alpha^{-1}\right)$, where, for any function $f$ on $G$, the translate $x \cdot f$ is defined by $(x \cdot f)(y)=f(y x)$. Thus, our definition means that the value of $(x, \alpha) \cdot w$ at $y$ is $w\left(\alpha^{-1}(y x)\right)$. In order to verify that this is indeed a representation of $G \rtimes J$ on $W$, it suffices to check that, compatibly with $\alpha x=\alpha(x) \alpha$ (in $G \rtimes J$ ), one has $\alpha \cdot(x \cdot w)=\alpha(x) \cdot(\alpha \cdot w)$. Evidently, this representation is analytic, and its restriction to $G$ is faithful.

In order to obtain a representation of $G \rtimes A$ whose restriction to $G$ is faithful, we use the ordinary group algebra $F[G \rtimes A]$, where $F$ is the field of real numbers or the field of complex numbers. We form the tensor product $F[G \times A] \otimes_{F[G \rtimes J]} W$ and let $G \rtimes A$ act via multiplication on the left factor. As a vector space, this module is the direct sum of $[A: J]$ copies of $W$, and thus is finite-dimensional. Since $A_{1} \subset J$, it is clear that this representation is analytic.

Finally, we make $\mathscr{L}(G)$ into a $G \rtimes A$ - module via the canonical homomorphisms $G \rtimes A \rightarrow A \rightarrow B$, so that the kernel of this representation of $G \times A$ on $\mathscr{L}(G)$ is precisely $G$. The direct sum of the two representations we have constructed satisfies the requirements of our theorem.

The simplest example of a faithfully representable analytic group $G$ such that $G \rtimes A$ is not faithfully representable is the direct product $\boldsymbol{R} \times T$ of the additive group $\boldsymbol{R}$ of real numbers and the multiplicative group $T$ of complex numbers of absolute value 1. For every real number $a$, this group has an analytic automorphism $a^{*}$, where $a^{*}(r, u)=(r, \exp (i a r) u)$. If $S$ is the space of representative functions associated with a representation of $G$ that is not trivial on $T$, it is easy to see that the space spanned by the functions $f \circ a^{*}$ with $f$ in $S$ and $a$ in $\boldsymbol{R}$ is not finite-dimensional.

We may summarize our results as follows.

Summary. Let $K$ be a real or complex Lie group having a semidirect product decomposition $G \rtimes H$, where $G$ is connected and faithfully representable, and the maximum nilpotent normal analy- 
tic subgroup $N$ of $G$ is simply connected. There is a finite-dimensional analytic representation of $K$ whose restriction to $G$ is faithful and whose restriction to $N$ is unipotent. If $H$ is faithfully representable there is a faithful such representation.

Proof. Let $A$ denote the group of all analytic automorphisms of $G$. The semidirect product decomposition of $K$ defines an analytic homomorphism $\eta: H \rightarrow A$ and hence an analytic homomorphism $\eta^{*}$ : $K \rightarrow G \rtimes A$. If $\rho$ is a representation of $G \times A$ as obtained in the theorem, then $\rho \circ \eta^{*}$ clearly satisfies the requirements of the first statement of the summary. The second statement then follows immediately.

\section{REFERENCE}

1. G. Hochschild, The structure of Lie groups, Holden-Day, San Francisco, 1965.

Received February 25, 1978.

UNIVERSITY OF CALIFORNIA

BERKELEY, CA 94720 


\title{
PACIFIC JOURNAL OF MATHEMATICS
}

\section{EDITORS}

RICHARD ARENS (Managing Editor)

University of California

Los Angeles, CA 90024

Charles W. Curtis

University of Oregon

Eugene, OR 97403

C. C. MOORE

University of California

Berkeley, CA 94720

\section{J. DUGUNDJI}

Department of Mathematics University of Southern California Los Angeles, CA 90007

R. Finn and J. Milgram

Stanford University

Stanford, CA 94305

\section{ASSOCIATE EDITORS}
E. F. BECKENBACH
B. H. NeumanN
F. WOLF
K. YOSHIDA

\section{SUPPORTING INSTITUTIONS}

\author{
UNIVERSITY OF BRITISH COLUMBIA \\ CALIFORNIA INSTITUTE OF TECHNOLOGY \\ UNIVERSITY OF CALIFORNIA \\ MONTANA STATE UNIVERSITY \\ UNIVERSITY OF NEVADA, RENO \\ NEW MEXICO STATE UNIVERSITY \\ OREGON STATE UNIVERSITY \\ UNIVERSITY OF OREGON
}

\author{
UNIVERSITY OF SOUTHERN CALIFORNIA \\ STANFORD UNIVERSITY \\ UNIVERSITY OF HAWAII \\ UNIVERSITY OF TOKYO \\ UNIVERSITY OF UTAH \\ WASHINGTON STATE UNIVERSITY \\ UNIVERSITY OF WASHINGTON
}

The Supporting Institutions listed above contribute to the cost of publication of this Journal, but they are not owners or publishers and have no responsibility for its content or policies.

Mathematical papers intended for publication in the Pacific Journal of Mathematics should be in typed form or offset-reproduced, (not dittoed), double spaced with large margins. Please do not use built up fractions in the text of the manuscript. However, you may use them in the displayed equations. Underline Greek letters in red, German in green, and script in blue. The first paragraph or two must be capable of being used separately as a synopsis of the entire paper. Items of the bibliography should not be cited there unless absolutely necessary, in which case they must be identified by author and journal, rather than by item number. Manuscripts, in triplicate, may be sent to any one of the editors. Please classify according to the scheme of Math. Reviews, Index to Vol. 39. All other communications should be addressed to the managing editor, or Elaine Barth, University of California, Los Angeles, California, 90024.

50 reprints to each author are provided free for each article, only if page charges have been substantially paid. Additional copies may be obtained at cost in multiples of 50 .

The Pacific Journal of Mathematics is issued monthly as of January 1966. Regular subscription rate: $\$ 72.00$ a year (6 Vols., 12 issues). Special rate: $\$ 36.00$ a year to individual members of supporting institutions.

Subscriptions, orders for numbers issued in the last three calendar years, and changes of address should be sent to Pacific Journal of Mathematics, P.O. Box 969, Carmel Valley, CA 93924, U.S.A. Older back numbers obtainable from Kraus Periodicals Co., Route 100, Millwood, NY 10546.

PUBLISHED BY PACIFIC JOURNAL OF MATHEMATICS, A NON-PROFIT CORPORATION

Printed at Kokusai Bunken Insatsusha (International Academic Printing Co., Ltd.). 8-8, 3-chome, Takadanobaba, Shinjuku-ku, Tokyo 160, Japan.

Copyright (C) 1978 by Pacific Journal of Mathematics Manufactured and first issued in Japan 


\section{Pacific Journal of Mathematics \\ Vol. 78, No. $2 \quad$ April, 1978}

Su-Shing Chen, Weak rigidity of compact negatively curved manifolds .... 273

Heinz Otto Cordes and D. A. Williams, An algebra of pseudodifferential operators with nonsmooth symbol ....................... 279

Herbert Paul Halpern, Normal expectations and integral decomposition of type III von Neumann algebras ......................... 291

G. Hochschild, On representing analytic groups with their

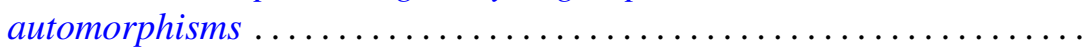

Dean G. Hoffman and David Anthony Klarner, Sets of integers closed under affine operators - the closure of finite sets ....................

Simeon Ivanov, On holomorphic relative inverses of operator-valued

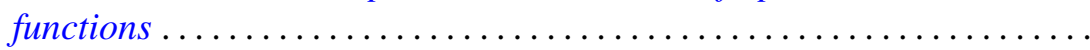

O. P. Juneja and M. L. Mogra, Radii of convexity for certain classes of

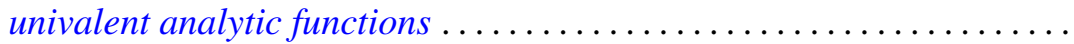

Hadi Kharaghani, The evolution of bounded linear functionals with application to invariant means.......................... 369

Jack W. Macki, A singular nonlinear boundary value problem .......... 375

A. W. Mason and Walter Wilson Stothers, Remarks on a theorem of $L$. Greenberg on the modular group ........................ 385

Kevin Mor McCrimmon, Peirce ideals in Jordan algebras . . . . . . . . . . . 397

John C. Morgan, II, On the absolute Baire property ................ 415

Gerard J. Murphy, Commutative non-Archimedean $C^{*}$-algebras ...

Masafumi Okumura, Submanifolds with L-flat normal connection of the

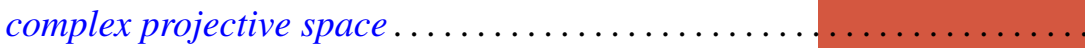

Chull Park and David Lee Skoug, Distribution estimates of barrier-crossing probabilities of the Yeh-Wiener process ...............

Irving Reiner, Invariants of integral representations ........

Phillip Schultz, The typeset and cotypeset of a rank 2 abelian group ..... 503

John Brendan Sullivan, Representations of Witt groups ....

Chia-Chi Tung, Equidistribution theory in higher dimensions . . . 